\title{
Non-slipping adhesive contact between mismatched elastic spheres: A model of adhesion mediated deformation sensor
}

\author{
Shaohua Chen ${ }^{\mathrm{a}}$, Huajian $\mathrm{Gao}^{\mathrm{b}, *}$ \\ ${ }^{a}$ LNM, Institute of Mechanics, Chinese Academy of Sciences, Beijing, 100080, China \\ ${ }^{\mathrm{b}}$ Division of Engineering, Brown University, Providence, RI 02912, USA
}

Received 10 January 2006; received in revised form 28 February 2006; accepted 6 March 2006

\begin{abstract}
A generalized JKR model is established for non-slipping adhesive contact between two dissimilar elastic spheres subjected to a pair of pulling forces and a mismatch strain. We discuss the full elastic solution to the problem as well as the so-called non-oscillatory solution in which tension and shear tractions along the contact interface is decoupled from each other. The model indicates that the mismatch strain has significant effect on the contact area and the pull-off process. Under a finite pulling force, a pair of adhering spheres is predicted to break apart spontaneously at a critical mismatch strain. This study suggests an adhesion mediated deformation sensing mechanism by which cells and molecules can detect mechanical signals in the environment via adhesive interactions.
\end{abstract}

(C) 2006 Elsevier Ltd. All rights reserved.

Keywords: Bio-adhesion; Adhesion and adhesives; Biological material; Contact mechanics

\section{Introduction}

There is accumulating biological evidence that cells actively sense and react to mechanical forces and deformation in the environment (Galbraith and Sheetz, 1998; Huang and Ingber, 1999; Geiger and Bershadsky, 2002). For example, fibroblasts on elastic substrates tend to orient in the direction of tensile strain (Haston et al., 1983) and

\footnotetext{
*Corresponding author. Tel.: + 14018632626.

E-mail address: Huajian_Gao@Brown.edu (H. Gao).
} 
migrate towards regions of larger stiffness (Lo et al., 2000). Similar response has also been reported for cells in hydrogels and for vascular smooth muscle cells on substrates with stiffness gradients (Wong et al., 2003). Almost every type of cells on cyclically stretched substrates has been found to align nearly perpendicular to the primary stretching direction, with cytoskeleton of the stretched cells remodeled into bundles of actin filaments oriented near the perpendicular direction (Dartsch and Hammerle, 1986; Dartsch and Betz, 1989; Iba and Sumpio, 1991; Neidlinger-Wilke et al., 1994). The cell orientation appeared to depend on the stretching magnitude: the larger the stretch, the more cells re-orient away from the stretching direction (Wang et al., 1995; Takemasa et al., 1997). Active mechanosensing allows cells to remodel their contacts and cytoskeleton to facilitate migration and re-orientation in response to mechanical forces in their environment. Lorz et al. (2000) have studied deformation and unbinding of weakly adhering giant vesicles under hydrodynamic shear forces. Bischofs and Schwarz (2003) have demonstrated that linear elasticity theory can be useful in explaining why typical cellular reaction to mechanical input seems to show preference for large effective stiffness.

At the molecular level, specific binding between protein molecules is believed to play an important role in cell adhesion and signal transduction. Protein molecules are deformable and can alter their conformations under mechanical forces. The conformation changes can in turn affect protein-protein and protein-DNA recognition, binding and unbinding (Bao, 2000; Zhu et al., 2000). Bao (2002) discussed that mechanical forces can cause a receptor molecule to deform, thereby altering the conformational match between the receptor and its ligand. In some cases, the effect of mechanical deformation may decrease the receptor-ligand binding and in others it may enhance the interactions by exposing the binding sites.

Contact mechanics theories have been increasingly used in helping understand bioadhesion mechanisms such as cell-cell contact (Chu et al., 2005), cells on stretched substrates (Chen and Gao, 2006), and hierarchical structures of Geckos and insects (Autumn et al., 2002; Artz et al., 2003; Persson, 2003; Gao and Yao, 2004; Glassmaker et al., 2004; Hui et al., 2004; Gao et al., 2005; Spolenak et al., 2005; Huber et al., 2005, Yao and Gao, 2006). These studies have significantly expanded the literature on classical adhesive contact mechanics represented by the models of JKR (Johnson, Kendall and Roberts, 1971), DMT (Derjaguin, Muller and Toporov, 1975), Maugis-Dugdale (Maugis, 1992) and others (Roberts and Thomas 1975; Muller et al., 1980; Greenwood and Johnson, 1981; Barquins, 1988; Carpick et al., 1996; Chaudhury et al. 1996; Greenwood, 1997; Johnson and Greenwood, 1997; Barthel, 1998; Robbe-Valloire and Barquins, 1998; Greenwood and Johnson, 1998; Kim et al., 1998; Morrow et al., 2003; Schwarz, 2003). Motivated by the question of whether contact mechanics model can be established to explain how cells and molecules sense mechanical deformation induced by environmental forces, in this paper we consider non-slipping adhesive contact between two dissimilar elastic bodies subjected to a pair of pulling forces and a mismatch strain induced by environmental forces such as changes in temperature and/or pressure. The model predicts that the mismatch strain has significant effect on both the contact area and the pull-off process. Under a finite pulling force (which may be interpreted as an effective force due to thermal or entropic forces), a pair of adhering particles is predicted to break apart spontaneously at a critical mismatch strain, thereby suggesting a mechanism by which cells and molecules can detect environmental changes via specific binding interactions. 
In most of the existing contact mechanics models, tangential tractions inside the contact region are usually neglected. A few exceptional cases have been studied in the past. The non-slipping Hertz contact problem was treated by Spence (1968a). Kendall (1975) investigated the effects of shrinkage stress on brittle interfacial failure of a bonded laminate. Savkoor and Briggs (1977) conducted experiments to show that an applied tangential force can reduce the area of contact between elastic solids. In the present model, the contact interface is assumed to be well bonded except that the contact edge is allowed to shift according to the thermodynamic equilibrium between elastic and surface energies. This boundary condition leads to a stress field with an oscillatory singularity near the contact edge, similar to that near an interfacial crack between dissimilar elastic materials. On the other hand, our analysis indicates that the oscillation has only negligible effect on the contact area and the pull-off process, hence can be neglected for all practical purposes. Our model can be regarded as a generalization of the JKR model to cases in which slip along the contact interface is not allowed and shear tractions become so important that interfacial fracture mechanics must be used to describe the elastic field near the contact edge.

In applying simple elastic models to biological phenomena, we caution that the mechanical properties of cells or proteins can be extremely complex (Howard, 2001; Bao, 2002). Although bio-adhesion would in principle be better described by viscoelastic contact models (Maugis and Barquins, 1978; Hui et al., 1998; Lin et al., 1999; Barthel and Haiat, 2002; Haiat et al., 2003), such solutions rely on detailed descriptions of biological systems that are often uncertain or unavailable. On the other hand, elastic models, with their limitations well understood, can provide useful insights into the basic principles of a complex problem. This viewpoint is adopted in the present study.

\section{Model}

Fig. 1 shows a schematic illustration of the problem under consideration. Two dissimilar elastic spheres are brought into adhesive contact and then subjected to the combined action of a pair of pulling forces with magnitude $F$ and a mismatch strain $\varepsilon_{m}$ induced by environmental forces such as changes in pressure and/or temperature. The contact interface is assumed to be perfectly bonded and the contact edge is allowed to shift in position according to thermodynamic equilibrium between elastic energy and surface energy. If the shear traction along the contact interface is neglected, as in the classical JKR model, one would predict that the mismatch strain $\varepsilon_{m}$ should have no influence on the contact area. In contrast, our model assumes no slipping along the contact interface so that the contact area will be influenced by both the pulling force $F$ and the mismatch strain $\varepsilon_{m}$. The contact radius $a$ is assumed small so that the deformation of each sphere can be approximated by that of an elastic half-space.

Our assumption that the contact area is perfectly bonded has been inspired by specific binding between receptors and their corresponding ligands in cell adhesion as well as specific sequence matching in adhesion between biomolecules. If there is one to one bonding between specific molecules, shear deformation along the contact interface would not be easily relaxed.

A pair of cylindrical coordinates $\left(r, \theta, z_{1}\right),\left(r, \theta, z_{2}\right)$ are placed at the center of the contact region of each sphere, with $z_{1}, z_{2}$ pointing into the corresponding body. The present contact problem has a number of features in common with an external circular interfacial 


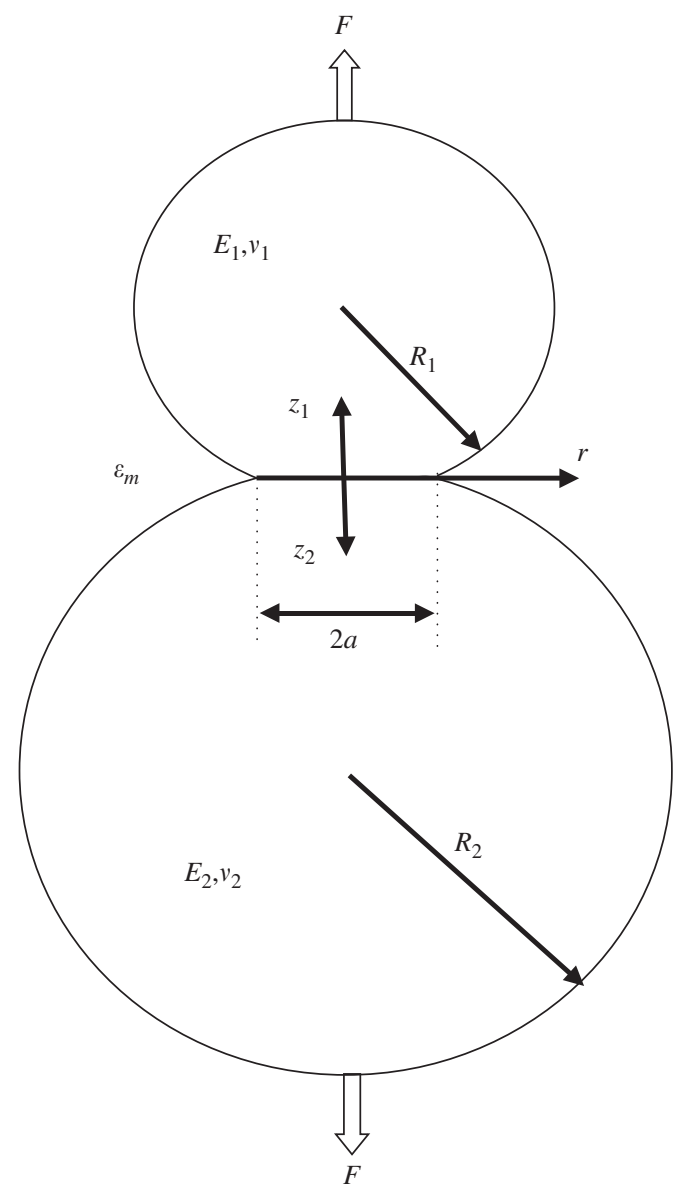

Fig. 1. Schematic illustration of two elastic spheres in non-slipping adhesive contact under a pair of pulling forces $F$ and a mismatch strain $\varepsilon_{m}$. The spheres have radii $R_{1}, R_{2}$, contact radius $a$ and elastic properties $\left(E_{1}, v_{1}\right),\left(E_{2}, v_{2}\right)$. A pair of cylindrical coordinates $\left(r, \theta, z_{1}\right),\left(r, \theta, z_{2}\right)$ are attached at the center of the contact region of each sphere, with $z_{1}, z_{2}$ pointing into the corresponding body.

crack, the energy release rate of which can be expressed as (Rice, 1965; Erdogan, 1965; Westmann, 1965)

$$
G=\frac{1}{\cosh ^{2} \pi \kappa} \frac{|K|^{2}}{2 E^{*}},
$$

where $\kappa$ is the so-called oscillation index to be defined in Eq. (23), $K$ is a complex valued stress intensity factor to be determined in Eq. (28) and $E^{*}$ is the combined Young's modulus

$$
\frac{1}{E^{*}}=\frac{1-v_{1}^{2}}{E_{1}}+\frac{1-v_{2}^{2}}{E_{2}}
$$

$E_{1}, v_{1}, E_{2}, v_{2}$ being the Young's moduli and Poisson's ratios of the two contacting objects, as shown in Fig. 1. 
The thermodynamic equilibrium between elastic and surface energies at the contact edge can be expressed in terms of Griffith's condition

$$
G=\Delta \gamma=\gamma_{1}+\gamma_{2}-2 \gamma_{12}
$$

where $\Delta \gamma$ is the work of adhesion, $\gamma_{1}, \gamma_{2}$ are the surface energies of the two contacting bodies and $\gamma_{12}$ is the energy of the contact interface.

\section{Analysis}

\subsection{General solution}

In order to formulate the axisymmetric contact problem described in Section 2, we first consider an elastic half space $(z \geqslant 0)$ subjected to axisymmetric normal and tangential tractions over a circular region of radius $a$ on the surface. The displacement and stress components inside the half space can be expressed as (Sneddon, 1951; Spence, 1968a)

$$
\begin{aligned}
& u_{r}(r, z)=\frac{\lambda+\mu}{\mu} \int_{0}^{\infty} \xi^{2} \frac{\mathrm{d} G}{\mathrm{~d} z} J_{1}(\xi r) \mathrm{d} \xi \\
& u_{z}(r, z)=\int_{0}^{\infty} \xi\left(\frac{\mathrm{d}^{2} G}{\mathrm{~d} z^{2}}-\frac{\lambda+2 \mu}{\mu} \xi^{2} G\right) J_{0}(\xi r) \mathrm{d} \xi \\
& \sigma_{r z}(r, z)=\int_{0}^{\infty} \xi^{2}\left[\lambda \frac{\mathrm{d}^{2} G}{\mathrm{~d} z^{2}}+(\lambda+2 \mu) \xi^{2} G\right] J_{1}(\xi r) \mathrm{d} \xi \\
& \sigma_{z z}(r, z)=\int_{0}^{\infty} \xi\left[(\lambda+2 \mu) \frac{\mathrm{d}^{3} G}{\mathrm{~d} z^{3}}-(3 \lambda+4 \mu) \xi^{2} \frac{\mathrm{d} G}{\mathrm{~d} z}\right] J_{0}(\xi r) \mathrm{d} \xi,
\end{aligned}
$$

where $\lambda$ and $\mu$ are Lame's elastic constants of the half space, $J_{0}(\rho t)$ and $J_{1}(\rho t)$ are Bessel functions, $G$ satisfies $\mathrm{d}^{4} G / \mathrm{d} z^{4}-2 \xi^{2} \mathrm{~d}^{2} G / \mathrm{d} z^{2}+\xi^{4} G=0$ with solution

$$
G(\xi, z)=[A(\xi)+z B(\xi)] \mathrm{e}^{-\xi z}
$$

for $z \geqslant 0, A(\xi)$ and $B(\xi)$ being two unknown functions to be determined from the boundary conditions.

Along $z=0$, letting $\xi=t / a, \rho=r / a$ and

$$
\begin{aligned}
& \frac{(\lambda+\mu) \xi^{3} A(\xi)+\mu \xi^{2} B(\xi)}{a^{2}}=-\mu g(t), \\
& \frac{(\lambda+\mu) \xi^{3} A(\xi)-\lambda \xi^{2} B(\xi)}{a^{2}}=-\mu h(t),
\end{aligned}
$$

allow the surface displacements $u_{z}, u_{r}$ and stresses $\sigma_{z z}, \sigma_{r z}$ to be expressed as

$$
\begin{aligned}
& u_{z}(\rho)=a \int_{0}^{\infty}[2(1-v) g(t)-(1-2 v) h(t)] J_{0}(\rho t) \mathrm{d} t, \\
& u_{r}(\rho)=a \int_{0}^{\infty}[2(1-v) h(t)-(1-2 v) g(t)] J_{1}(\rho t) \mathrm{d} t, \\
& \sigma_{z z}(\rho)=-2 \mu \int_{0}^{\infty} \operatorname{tg}(t) J_{0}(\rho t) \mathrm{d} t, \\
& \sigma_{r z}(\rho)=-2 \mu \int_{0}^{\infty} \operatorname{th}(t) J_{1}(\rho t) \mathrm{d} t .
\end{aligned}
$$


In this form, the unknown functions $g(t)$ and $h(t)$ correspond to the Hankel transforms of $\sigma_{z z}$ and $\sigma_{r z}$, respectively.

Now consider two elastic spheres that have been brought into non-slipping adhesive contact at a reference state. After the binding is formed, a pair of pulling forces $F$ and a mismatch strain $\varepsilon_{m}$ are imposed on the two spheres. This means that, if the interface is not bonded, the two spheres would undergo a relative tangential displacement

$$
\Delta u_{r}=u_{r 1}-u_{r 2}=\varepsilon_{m} a \rho,
$$

along the contact interface, where the subscripts " 1 " and " 2 " denote the upper and lower spheres, respectively. However, since the interface is bonded, this relative displacement can not occur and is instead compensated by elastic deformation in the two spheres. Such mismatch strain could be induced by, for example, a sudden change in pressure $\Delta p$ or in temperature $\Delta T$ in the environment, in which cases

$$
\varepsilon_{m}=\Delta p\left(\frac{1}{K_{1}}-\frac{1}{K_{2}}\right) \quad \text { or } \quad \varepsilon_{m}=\left(\alpha_{1}-\alpha_{2}\right) \cdot \Delta T,
$$

where $K_{1}$ and $K_{2}$ denote the bulk moduli, while $\alpha_{1}$ and $\alpha_{2}$ denote the thermal expansion coefficients, of the two spheres.

We adopt the usual parabolic approximation of contacting surfaces near the contact region where the normal surface displacements $u_{z 1}, u_{z 2}$, measured positive into each body, should satisfy

$$
\Delta u_{z}=u_{z 1}+u_{z 2}=\delta-\frac{a^{2}}{2 R} \rho^{2} .
$$

Here, $R$ is the combined radius $1 / R=1 / R_{1}+1 / R_{2}$ and $\delta$ is the relative displacement between the centers of the two objects (Johnson, 1985).

The continuity of normal and tangential tractions requires

$$
\sigma_{z z 1}=\sigma_{z z 2}=\sigma_{z z}, \quad \sigma_{r z 1}=-\sigma_{r z 2}=\sigma_{r z},
$$

where $\sigma_{z z 1}$ and $\sigma_{r z 1}$ denote the normal and tangential tractions on the surface of the upper sphere, and $\sigma_{z z 2}$ and $\sigma_{r z 2}$ those on the lower sphere. Note that the axes $z_{1}, z_{2}$ are defined such that they point separately into each of the two corresponding contacting bodies.

Making use of Eq. (9), one can express Eq. (13) as

$$
\mu_{1} g_{1}(t)=\mu_{2} g_{2}(t)=\bar{\mu} \bar{g}(t), \quad \mu_{1} h_{1}(t)=-\mu_{2} h_{2}(t)=\bar{\mu} \bar{h}(t),
$$

where

$$
\bar{\mu}=\frac{E^{*}}{4(1-\beta)}, \quad \beta=\frac{1}{2}\left\{\frac{\left(1-2 v_{1}\right)\left(1+v_{1}\right) / E_{1}-\left(1-2 v_{2}\right)\left(1+v_{2}\right) / E_{2}}{\left(1-v_{1}^{2}\right) / E_{1}+\left(1-v_{2}^{2}\right) / E_{2}}\right\},
$$

$\beta$ being one of Dundurs' constants (Durdurs, 1969) for the bimaterial system.

Combining Eqs. (8)-(10), (12) and (14) yields a set of coupled dual integral equations that govern the non-slipping adhesive contact problem,

$$
\sigma_{z z}(\rho)=-\frac{E^{*}}{2(1-\beta)} \int_{0}^{\infty} t \bar{g}(t) J_{0}(\rho t) \mathrm{d} t, \quad \sigma_{r z}(\rho)=-\frac{E^{*}}{2(1-\beta)} \int_{0}^{\infty} t \bar{h}(t) J_{1}(\rho t) \mathrm{d} t,
$$




$$
\Delta u_{z}(\rho)=\frac{a}{1-\beta} \int_{0}^{\infty}[\bar{g}(t)-\beta \bar{h}(t)] J_{0}(\rho t) \mathrm{d} t, \quad \Delta u_{r}(\rho)=\frac{a}{1-\beta} \int_{0}^{\infty}[\bar{h}(t)-\beta \bar{g}(t)] J_{1}(\rho t) \mathrm{d} t
$$

to be solved with the boundary conditions,

$$
\begin{aligned}
& \Delta u_{r}(\rho)=\varepsilon_{m} a \rho, \quad \Delta u_{z}(\rho)=\delta-\frac{a^{2}}{2 R} \rho^{2}, \quad(\rho \leqslant 1) \\
& \sigma_{r z}(\rho)=\sigma_{z z}(\rho)=0, \quad(\rho>1)
\end{aligned}
$$

and

$$
F=2 \pi a^{2} \int_{0}^{1} \rho \sigma_{z z}(\rho) \mathrm{d} \rho .
$$

The solution to the governing equations (16)-(20) can be obtained by adapting a Wiener-Hopf method developed by Spence (1968a,b) for treating axisymmetric nonslipping Hertzian contact problems. The calculations are lengthy but the methodology is quite standard. Here, we skip all the details and present only the final solution. The interfacial tractions in the contact region have the solution,

$$
\begin{aligned}
& \sigma_{z z}(\rho)=\frac{E^{*}}{2 \pi \rho \sqrt{1-\beta^{2}}} \frac{\mathrm{d}}{\mathrm{d} \rho} \int_{\rho}^{1} \frac{w\left[R_{0}(w) \cos \kappa \theta+w R_{1}(w) \sin \kappa \theta\right]}{\sqrt{w^{2}-\rho^{2}}} \mathrm{~d} w, \\
& \sigma_{r z}(\rho)=\frac{E^{*}}{2 \pi \sqrt{1-\beta^{2}}} \frac{\mathrm{d}}{\mathrm{d} \rho} \int_{\rho}^{1} \frac{\left[R_{0}(w) \sin \kappa \theta-w R_{1}(w) \cos \kappa \theta\right]}{\sqrt{w^{2}-\rho^{2}}} \mathrm{~d} w,
\end{aligned}
$$

where

$$
\kappa=\frac{1}{2 \pi} \ln \frac{1+\beta}{1-\beta}
$$

is the so-called oscillation index for a bimaterial interfacial crack and

$$
\begin{aligned}
& \theta(w)=\ln \left|\frac{w+1}{w-1}\right|=\left\{\begin{array}{ll}
2 \tanh ^{-1} w & |w|<1, \\
2 \operatorname{coth}^{-1} w & |w|>1,
\end{array} \quad \theta(w)=-\theta(-w),\right. \\
& R_{0}(w)=\frac{2 \delta}{a}+8 \kappa \varepsilon_{m}+\frac{4 a \kappa^{2}}{R}-\frac{2 a w^{2}}{R}, \\
& R_{1}(w)=-4 \varepsilon_{m}-\frac{4 a \kappa}{R} .
\end{aligned}
$$

The force balance equation (20) leads to

$$
\begin{aligned}
\frac{\delta}{a}= & \frac{-F \sqrt{1-\beta^{2}}}{2 E^{*} a^{2} \int_{0}^{1} \cos \kappa \theta \mathrm{d} w}+2 \varepsilon_{m}\left[\frac{\int_{0}^{1} w \sin \kappa \theta \mathrm{d} w}{\int_{0}^{1} \cos \kappa \theta \mathrm{d} w}-2 \kappa\right] \\
& +\frac{2 \kappa a}{R} \frac{\int_{0}^{1} w \sin \kappa \theta \mathrm{d} w}{\int_{0}^{1} \cos \kappa \theta \mathrm{d} w}-\frac{2 \kappa^{2} a}{R}+\frac{a}{R} \frac{\int_{0}^{1} w^{2} \cos \kappa \theta \mathrm{d} w}{\int_{0}^{1} \cos \kappa \theta \mathrm{d} w}
\end{aligned}
$$


It can be shown (details omitted here) that the interfacial tractions in Eqs. (21) and (22) exhibit oscillatory singular behaviors near the contact edge with a complex valued stress intensity factor

$$
\begin{aligned}
K & =\lim _{\rho \rightarrow 1} \sqrt{2 \pi} a^{1 / 2+i \kappa}(1-\rho)^{1 / 2+i \kappa}\left[\sigma_{z z}(\rho)+\mathrm{i} \sigma_{r z}(\rho)\right] \\
& =\frac{E^{*} \sqrt{a}(2 \kappa i-1)(2 a)^{\kappa i}}{2 \sqrt{\pi\left(1-\beta^{2}\right)}}\left[R_{0}(1)-\mathrm{i} R_{1}(1)\right] \sum_{n=0}^{\infty} \frac{d_{n}}{2+2 n-2 \kappa i},
\end{aligned}
$$

where $\mathrm{i}=\sqrt{-1}$ and

$$
d_{0}=1, \quad d_{n}=\frac{(2 n-1) ! !}{2 n ! !} \quad(n>1),
$$

are the coefficients of the series expansion

$$
(1-x)^{-1 / 2}=\sum_{n=0}^{\infty} d_{n} x^{n} .
$$

Calculating the energy release rate by inserting Eq. (28) into Eq. (1), and then applying the Griffith energy balance in Eq. (3) yield the following relation:

$$
\begin{aligned}
& \left(\frac{-F \sqrt{1-\beta^{2}}}{E^{*} a^{2} \int_{0}^{1} \cos \kappa \theta \mathrm{d} w}+\left(4 \varepsilon_{m}+\frac{4 \kappa a}{R}\right) \frac{\int_{0}^{1} w \sin \kappa \theta \mathrm{d} w}{\int_{0}^{1} \cos \kappa \theta \mathrm{d} w}-\frac{2 a}{R}+\frac{2 a}{R} \frac{\int_{0}^{1} w^{2} \cos \kappa \theta \mathrm{d} w}{\int_{0}^{1} \cos \kappa \theta \mathrm{d} w}\right)^{2} \\
& +\left(4 \varepsilon_{m}+\frac{4 \kappa a}{R}\right)^{2}=\frac{8 \pi \Delta \gamma}{E^{*} a\left(1+4 \kappa^{2}\right)}\left|\sum_{n=0}^{\infty} \frac{d_{n}}{2+2 n-2 \kappa i}\right|^{-2},
\end{aligned}
$$

among the contact radius $a$, the mismatch strain $\varepsilon_{m}$ and the pulling force $F$.

\subsection{The non-oscillatory solution}

In a recent study on two-dimensional non-slipping adhesive contact problem (Chen and Gao, 2006), we found that the Dundurs' constant $\beta$ has negligible effects on the contact radius and the pull-off process. In the case of $\beta=0$, the governing equations (16) and (17) become

$$
\begin{aligned}
& \sigma_{z z}(\rho)=-\frac{E^{*}}{2} \int_{0}^{\infty} t \bar{g}(t) J_{0}(\rho t) \mathrm{d} t, \quad \Delta u_{z}(\rho)=a \int_{0}^{\infty} \bar{g}(t) J_{0}(\rho t) \mathrm{d} t, \\
& \sigma_{r z}(\rho)=-\frac{E^{*}}{2} \int_{0}^{\infty} t \bar{h}(t) J_{1}(\rho t) \mathrm{d} t, \quad \Delta u_{r}(\rho)=a \int_{0}^{\infty} \bar{h}(t) J_{1}(\rho t) \mathrm{d} t,
\end{aligned}
$$

where the normal and shear tractions along the contact interface are decoupled from each other. The boundary conditions remain the same as those given in Eqs. (18)-(20). In this case, the stress field is no longer oscillatory near the contact edge, the oscillation index vanishes, i.e. $\kappa=0$ and the corresponding non-oscillatory solutions is much simpler than the fully coupled solutions given in Eqs. (21)-(31). 
The non-oscillatory solutions to the governing equations (32) and (33) and boundary conditions (18)-(20) are

$$
\begin{aligned}
& \sigma_{z z}(\rho)=-\frac{2 E^{*} a \sqrt{1-\rho^{2}}}{\pi R}+\frac{E^{*}}{\pi}\left[\frac{2 a}{3 R}+\frac{F}{2 E^{*} a^{2}}\right]\left(1-\rho^{2}\right)^{-1 / 2}, \\
& \sigma_{r z}(\rho)=\frac{-2 E^{*} \varepsilon_{m}}{\pi}\left(1-\rho^{2}\right)^{-1 / 2}
\end{aligned}
$$

Eq. (27) is reduced to

$$
\frac{\delta}{a}=\frac{a}{3 R}-\frac{F}{2 E^{*} a^{2}}
$$

In the non-oscillatory case, the complex valued stress intensity factor in Eqs. (1) and (28) becomes decoupled as $K=K_{\mathrm{I}}+\mathrm{i} K_{\mathrm{II}}$, where

$$
K_{\mathrm{I}}=\lim _{\rho \rightarrow 1} \sqrt{2 \pi a}(1-\rho)^{1 / 2} \sigma_{z z}(\rho)=\frac{2 E^{*} a^{3 / 2}}{3 \sqrt{\pi} R}+\frac{F}{2 \sqrt{\pi} a^{3 / 2}}
$$

is the mode I stress intensity factor and

$$
K_{\mathrm{II}}=\lim _{\rho \rightarrow 1} \sqrt{2 \pi a}(1-\rho)^{1 / 2} \sigma_{r z}(\rho)=-2 E^{*} \varepsilon_{m} \sqrt{\frac{a}{\pi}}
$$

is the mode II stress intensity factor. The mode I solution of Eq. (37) is consistent with that given by the JKR model (Johnson et al., 1971) and the mode II solution of Eq. (38) is consistent with a solution derived by Gao (1990) using a different method. Inserting Eqs. (37) and (38) into the Griffith energy balance for $\kappa=0$,

$$
G=\frac{\left(K_{\mathrm{I}}^{2}+K_{\mathrm{II}}^{2}\right)}{2 E^{*}}=\Delta \gamma
$$

results in an equation

$$
a^{3}+9 R^{2} \varepsilon_{m}^{2} a-\frac{9 \Delta \gamma \pi R^{2}}{2 E^{*}}+\frac{9 R^{2} F^{2}}{16 E^{* 2} a^{3}}+\frac{3 F R}{2 E^{*}}=0,
$$

which allows the contact radius $a$ to be determined as a function of the mismatch strain $\varepsilon_{m}$ and the pulling force $F$. When $\varepsilon_{m}=0$, the non-oscillatory solution of $a$ is found to be identical to that of the JKR model (Johnson et al., 1971)

$$
\left.a\right|_{\varepsilon_{m}=0}=a_{J K R}=\left\{\frac{3 R}{4 E^{*}}\left[-F+3 \pi R \Delta \gamma+\sqrt{-6 \pi R \Delta \gamma F+(3 \pi R \Delta \gamma)^{2}}\right]\right\}^{1 / 3} .
$$

Eq. (40) can be expressed in a normalized form as

$$
\hat{a}^{3}+9 \hat{R}^{2} \varepsilon_{m}^{2} \hat{a}-1+\hat{F}^{2} \hat{a}^{-3}-\hat{F}^{2}=0,
$$

where

$$
\hat{a}=a / a_{J K R}, \quad \hat{R}=\frac{R}{a_{J K R}}, \quad \hat{F}=\frac{3 F R}{4 E^{*} a_{J K R}^{3}} .
$$

When $F=0$, Eq. (42) becomes

$$
\hat{a}^{3}+9 \hat{R}^{2} \varepsilon_{m}^{2} \hat{a}-1=0,
$$


which has the explicit solution

$$
a=a_{J K R}\left[\left(\frac{1}{2}+\sqrt{\frac{1}{4}+\frac{729 \hat{R}^{6} \varepsilon_{m}^{6}}{27}}\right)^{1 / 3}-3 \hat{R}^{2} \varepsilon_{m}^{2}\left(\frac{1}{2}+\sqrt{\frac{1}{4}+\frac{729 \hat{R}^{6} \varepsilon_{m}^{6}}{27}}\right)^{-1 / 3}\right]
$$

where

$$
a_{J K R}=\left(\frac{9 \pi R^{2} \Delta \gamma}{2 E^{*}}\right)^{1 / 3}
$$

Alternatively, Eq. (40) can also be used to express the pulling force as a function of contact area and mismatch strain as

$$
\tilde{F}=-\frac{4}{3} \tilde{E} \tilde{a}^{3}+\frac{1}{2} \sqrt{32 \pi \tilde{E} \tilde{a}^{3}-64 \varepsilon_{m}^{2} \tilde{E}^{2} \tilde{a}^{4}}
$$

where

$$
\tilde{a}=\frac{a}{R}, \quad \tilde{E}=\frac{E^{*} R}{\Delta \gamma}, \quad \tilde{F}=\frac{F}{\Delta \gamma R} .
$$

\section{Discussions}

\subsection{Non-slipping adhesive contact without a mismatch strain: the JKR model}

The case without a mismatch strain $\varepsilon_{m}=0$ shall be referred to as the non-slipping JKR model. In this case, we can compare the contact radius predicted by the full elastic solution and the JKR solution in Eq. (41) is

$$
\left(\frac{a_{0}}{a_{J K R}}\right)^{3}=\frac{\frac{-F A_{2}}{3 \pi R \Delta \gamma}+A_{3}+\sqrt{\left(A_{2}^{2}-A_{1} A_{4}\right)\left(\frac{F}{3 \pi R \Delta \gamma}\right)^{2}+A_{3}^{2}-\frac{2 F A_{2} A_{3}}{3 \pi R \Delta \gamma}}}{A_{1}\left[\frac{-F}{3 \pi R \Delta \gamma}+1+\sqrt{\frac{-2 F}{3 \pi R \Delta \gamma}+1}\right]},
$$

where

$$
\begin{aligned}
& A_{1}=\frac{9}{4}\left(\frac{2 \kappa \int_{0}^{1} w \sin \kappa \theta \mathrm{d} w}{\int_{0}^{1} \cos \kappa \theta \mathrm{d} w}-1+\frac{\int_{0}^{1} w^{2} \cos \kappa \theta \mathrm{d} w}{\int_{0}^{1} \cos \kappa \theta \mathrm{d} w}\right)^{2}+9 \kappa^{2} \\
& A_{2}=\frac{-3 \sqrt{1-\beta^{2}}}{2 \int_{0}^{1} \cos \kappa \theta \mathrm{d} w}\left(\frac{2 \kappa \int_{0}^{1} w \sin \kappa \theta \mathrm{d} w}{\int_{0}^{1} \cos \kappa \theta \mathrm{d} w}-1+\frac{\int_{0}^{1} w^{2} \cos \kappa \theta \mathrm{d} w}{\int_{0}^{1} \cos \kappa \theta \mathrm{d} w}\right) \\
& A_{3}=\frac{1}{\left(1+4 \kappa^{2}\right)\left|\sum_{n=0}^{\infty} d_{n} /(2+2 n-2 \kappa i)\right|^{2}}, \quad A_{4}=\frac{1-\beta^{2}}{\left(\int_{0}^{1} \cos \kappa \theta \mathrm{d} w\right)^{2}} .
\end{aligned}
$$

In principle, the ratio $a_{0} / a_{J K R}$ depends on the non-dimensional parameter $F /(\pi \mathrm{R} \Delta \gamma)$ and Dundurs' parameter $\beta$. However, numerical evaluation of Eq. (49), as plotted in Fig. 2, indicates that $a_{0} / a_{J K R}$ is close to 1 for $-\frac{1}{4}<\beta<\frac{1}{4}$, the range for most materials, at different values of $F /(\pi R \Delta \gamma)$, with maximum difference of about $2 \%$. The influence of $F /(\pi R \Delta \gamma)$ on 


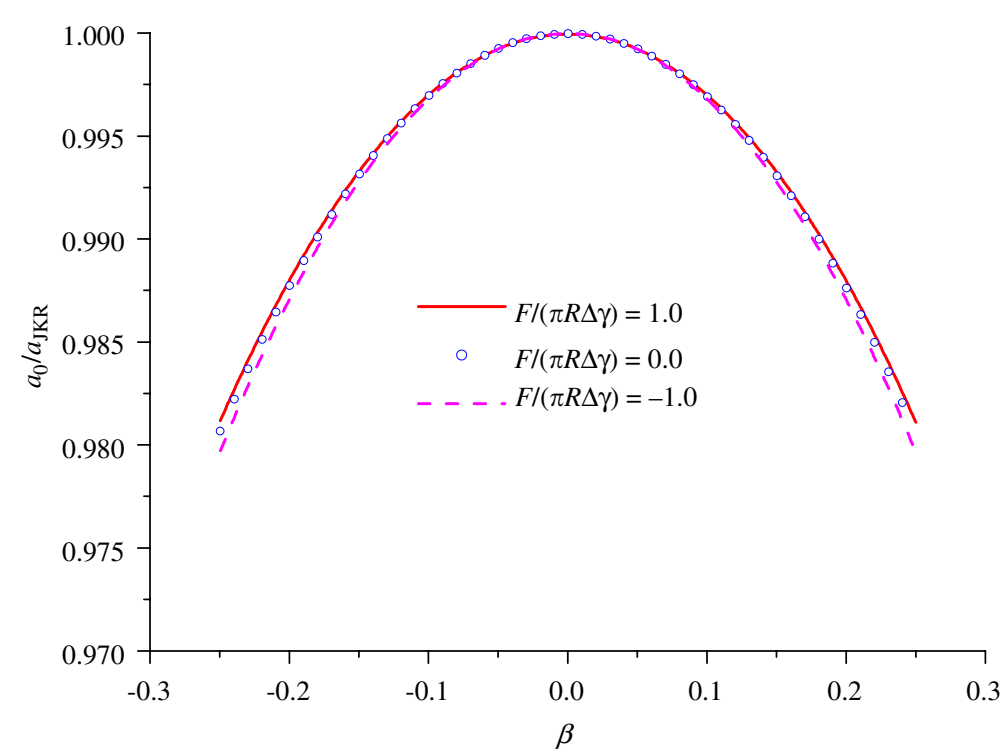

Fig. 2. Effect of Dundurs' parameter $\beta$ on the contact radius $a_{0}$ in the absence of a mismatch strain $\left(\varepsilon_{m}=0\right)$. The JKR solution $a_{J K R}$ corresponds to the non-oscillatory solution when $\beta$ is set to zero. The ratio between $a_{0}$ and $a_{J K R}$ is plotted as a function of $\beta$ for different values of the normalized pulling force $F /(\pi R \Delta \gamma)$, showing that the difference between $a_{0}$ and $a_{J K R}$ is less than $2 \%$ for the parameters ranges considered.

$a_{0} / a_{J K R}$ also seems quite small, as shown for three representative cases in Fig. 2. This result indicates that the coupling between normal and shear tractions is practically negligible (Johnson, 1985). The non-oscillatory solution, which in this case corresponds to the classical JKR model, serves as a good approximate solution to the non-slipping adhesive contact problem. For practical purposes, we can assume $a_{0} \approx a_{J K R}$.

\subsection{Non-slipping adhesive contact with a mismatch strain}

The shear tractions across the contact interface cannot be ignored in the presence of a mismatch strain. In this case, the shear tractions play a very important role. Interestingly, the non-oscillatory solution still remains a valid approximation, as discussed below.

Normalizing the contact radius $a$ in Eq. (31) with $a_{0} \approx a_{J K R}$ in Eq. (49) gives

$$
B_{1} \varepsilon_{m}^{2}+B_{2} \varepsilon_{m}+B_{3}=0,
$$

where

$$
\begin{aligned}
B_{1}= & 16 \hat{a} \hat{R}^{2}\left[1+\left(\int_{0}^{1} w \sin \kappa \theta \mathrm{d} w\right)^{2}\left(\int_{0}^{1} \cos \kappa \theta \mathrm{d} w\right)^{-2}\right] \\
B_{2}= & \frac{-32 \sqrt{1-\beta^{2}} \hat{R} \hat{F}}{3 \hat{a}} \int_{0}^{1} w \sin \kappa \theta \mathrm{d} w\left(\int_{0}^{1} \cos \kappa \theta \mathrm{d} w\right)^{-2} \\
& +16 \hat{a}^{2} \hat{R}\left[2 \kappa+\frac{\int_{0}^{1} w \sin \kappa \theta \mathrm{d} w}{\int_{0}^{1} \cos \kappa \theta \mathrm{d} w}\left(\frac{2 \kappa \int_{0}^{1} w \sin \kappa \theta \mathrm{d} w}{\int_{0}^{1} \cos \kappa \theta \mathrm{d} w}-1+\frac{\int_{0}^{1} w^{2} \cos \kappa \theta \mathrm{d} w}{\int_{0}^{1} \cos \kappa \theta \mathrm{d} w}\right)\right],
\end{aligned}
$$




$$
\begin{aligned}
B_{3}= & 4\left(\hat{a}^{3}-1\right)\left(\frac{2 \kappa \int_{0}^{1} w \sin \kappa \theta \mathrm{d} w}{\int_{0}^{1} \cos \kappa \theta \mathrm{d} w}-1+\frac{\int_{0}^{1} w^{2} \cos \kappa \theta \mathrm{d} w}{\int_{0}^{1} \cos \kappa \theta \mathrm{d} w}\right)^{2}+16 \kappa^{2}\left(\hat{a}^{3}-1\right) \\
& +\frac{16 \hat{F}^{2}\left(1-\beta^{2}\right)}{9\left(\int_{0}^{1} \cos \kappa \theta \mathrm{d} w\right)^{2}}\left[\frac{1}{\hat{a}^{3}}-1\right]
\end{aligned}
$$

and

$$
\hat{a}=a / a_{0} \approx a / a_{J K R}, \quad \hat{R}=R / a_{0} \approx R / a_{J K R}, \quad \hat{F}=\frac{3 F R}{4 E^{*} a_{0}^{3}} \approx \frac{3 F R}{4 E^{*} a_{J K R}^{3}} .
$$

Numerical calculations show that the influence of Dundurs' parameter $\beta$ on the above solution is negligible. Fig. 3 plots $a / a_{J K R}$ as a function of $\varepsilon_{m}$ for five different values of $\beta$ under two representative choices of $\hat{R}=R / a_{J K R}$ and $\hat{F}=3 F R / 4 E^{*} a_{J K R}^{3}$. In all cases, the effect of $\beta$ is quite small and can be neglected for practical purposes. Therefore, the nonoscillatory solutions in Eqs. (34)-(48) can serve as an approximate solution to the nonslipping adhesive contact problem between two dissimilar elastic spheres.

The non-oscillatory solution in (42) and (43) shows that the normalized contact radius $a / a_{J K R}$ depends on the mismatch strain $\varepsilon_{m}$ and the normalized radius $\hat{R}=R / a_{J K R}$ only through the combined parameter $\hat{R} \varepsilon_{m}$. In addition, $a / a_{J K R}$ depends on the normalized pulling force $\hat{F}=3 F R / 4 E^{*} a_{J K R}^{3}$. Fig. 4 plots $a / a_{J K R}$ as a function of $\hat{R} \varepsilon_{m}$ for different values of $\hat{F}$. In the absence of the pulling force $\hat{F}$, the contact radius varies smoothly with the mismatch strain $\varepsilon_{m}$ and exhibits three distinct regimes of behaviors characterized by two threshold strains: (i) the contact size is hardly affected by $\varepsilon_{m}$ when $\varepsilon_{m}$ is below the first threshold level; (ii) the contact radius decreases quickly with $\varepsilon_{m}$ as $\varepsilon_{m}$ increases between the two threshold levels; (iii) the contact size approaches zero when the substrate strain exceeds the second threshold level. This result is qualitatively similar to our previous study on the two dimensional non-slipping adhesive contact problem of an elastic cylinder on a stretched substrate (Chen and Gao, 2006).

More interestingly, under a finite pulling force $\hat{F}$ (which can be interpreted as an effective force due to thermal or entropic forces), there exists a critical mismatch strain, shown by the critical values of $\hat{R} \varepsilon_{m}$ in Fig. 4, at which a pair of adhering spheres is predicted to break apart spontaneously. The larger the value of $\hat{F}$, the smaller the critical value of $\hat{R} \varepsilon_{m}$. If thermal fluctuations are considered as an effective pulling force on two adhering molecules or cells, our result immediately suggests a mechanism by which cells or molecules can sense mechanical deformation: the number density of molecules or cells in contact may sensitively depend on the mismatch strain induced by environment forces. For example, changes in temperature or pressure or $\mathrm{PH}$ values would induce mismatch strains between adhering molecules or cells, which could then influence the behaviors of receptorligand bonds, thereby transmitting mechanical signals via adhesive interactions.

\subsection{The influence of mismatch strain on the pull-off process}

In Eq. (47), we have expressed the normalized force $\tilde{F}=F /(\Delta \gamma R)$ as a function of the mismatch strain $\varepsilon_{m}$, the normalized contact radius $\tilde{a}=a / R$ and the normalized Young's modulus $\tilde{E}=E^{*} R / \Delta \gamma$. Fig. 5 plots $\tilde{F}$ as a function of $\tilde{a}$ for three different values of the mismatch strain when $\tilde{E}=10000$. For a given $\varepsilon_{m}$, the contact radius decreases as the force 


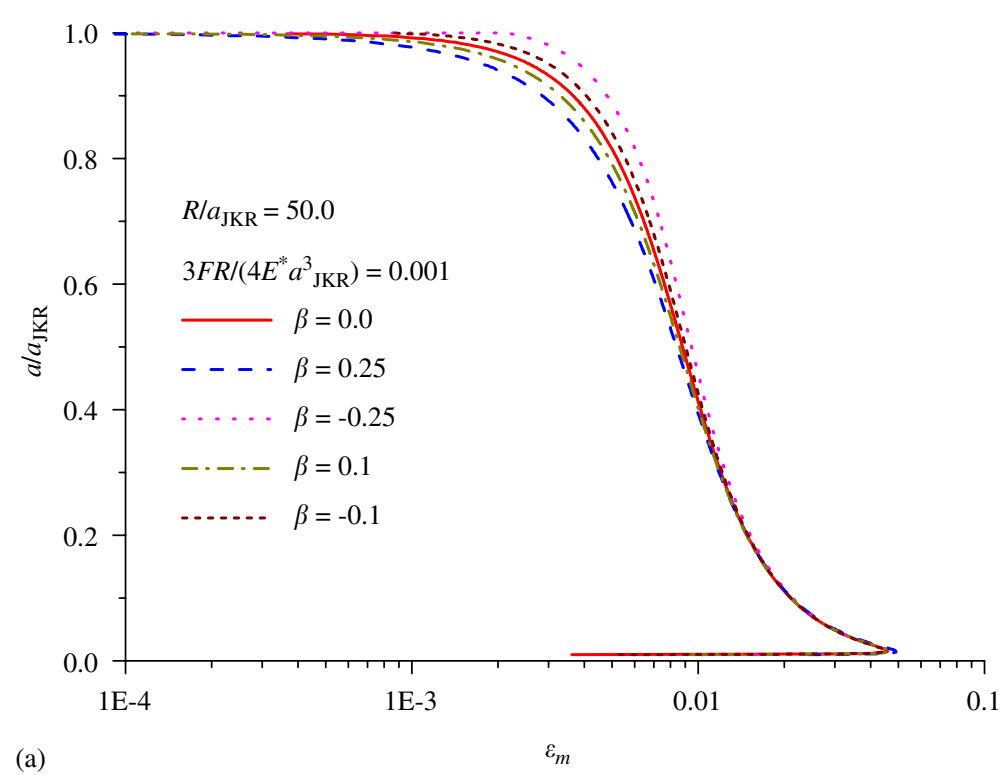

(a)

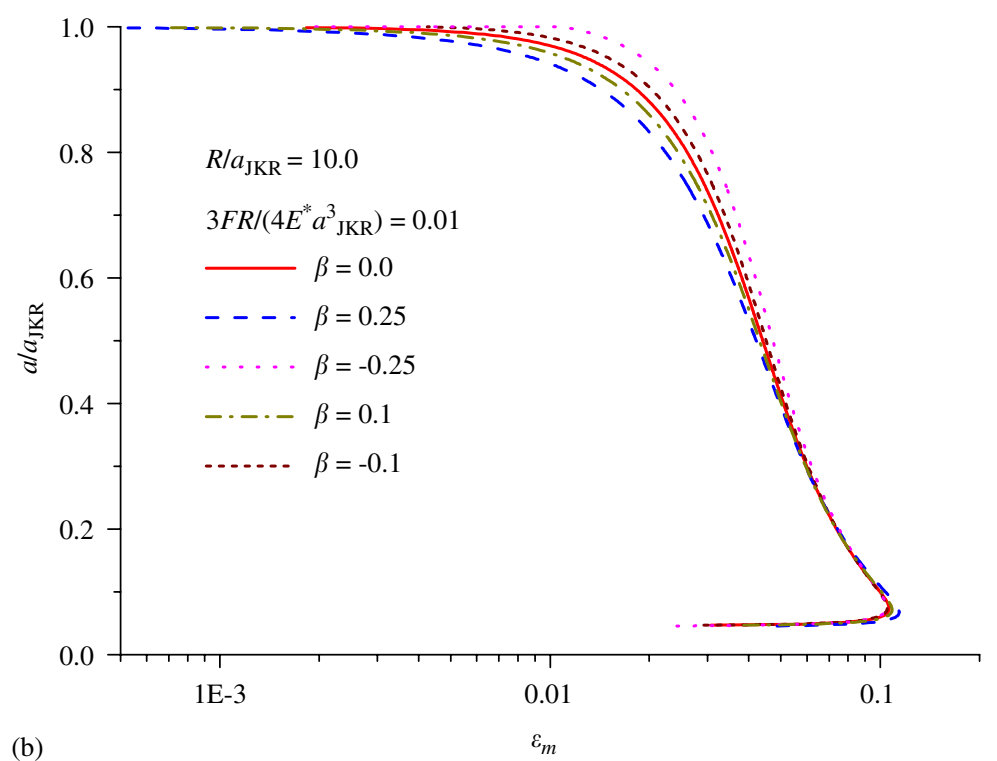

Fig. 3. Effect of Dundurs' parameter $\beta$ on the contact radius $a$ in the presence of a mismatch strain $\varepsilon_{m}$. The normalized contact radius $a / a_{J K R}$ is plotted as a function of $\varepsilon_{m}$ for different values of $\beta$ and parameters choices of (a) $\hat{R}=50.0, \hat{F}=0.001$ and (b) $\hat{R}=10.0, \hat{F}=0.01$, where $\hat{R}=R / a_{J K R}$ and $\hat{F}=3 F R /\left(4 E^{*} a_{J K R}^{3}\right)$. The results indicate that $\beta$ has negligible effect so that the non-oscillatory solution can be used as a good approximate solution.

$\tilde{F}$ changes from compression to tension until the two spheres are pulled off at a critical force. The case $\varepsilon_{m}=0$ corresponds to the classical JKR model. The results in Fig. 5 indicate that the mismatch strain has significant effects on the pull-off process. The pull-off 


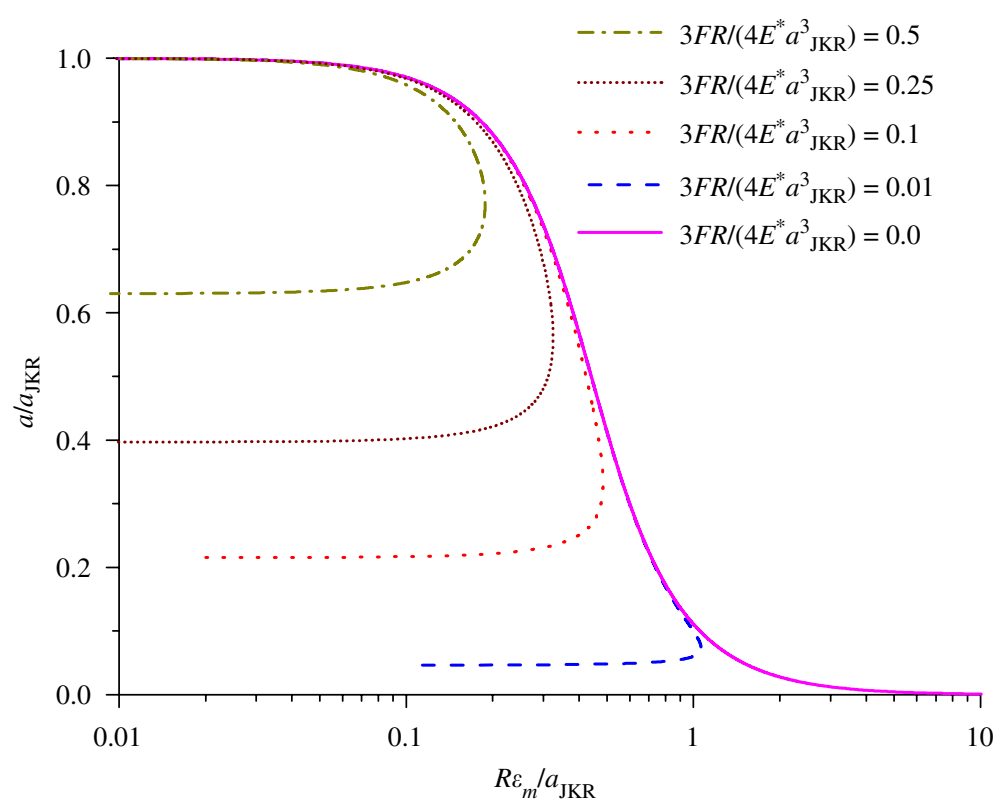

Fig. 4. Spontaneous detachment of adhering spheres at a critical mismatch strain. The normalized contact radius $a / a_{J K R}$ is plotted as a function of $\hat{R} \varepsilon_{m}\left(\hat{R}=R / a_{J K R}\right)$ according to Eqs. (42) and (43). Under a finite pulling force, the contact radius decreases with increasing $\varepsilon_{m}$ and an instability occurs at a critical $\varepsilon_{m}$.

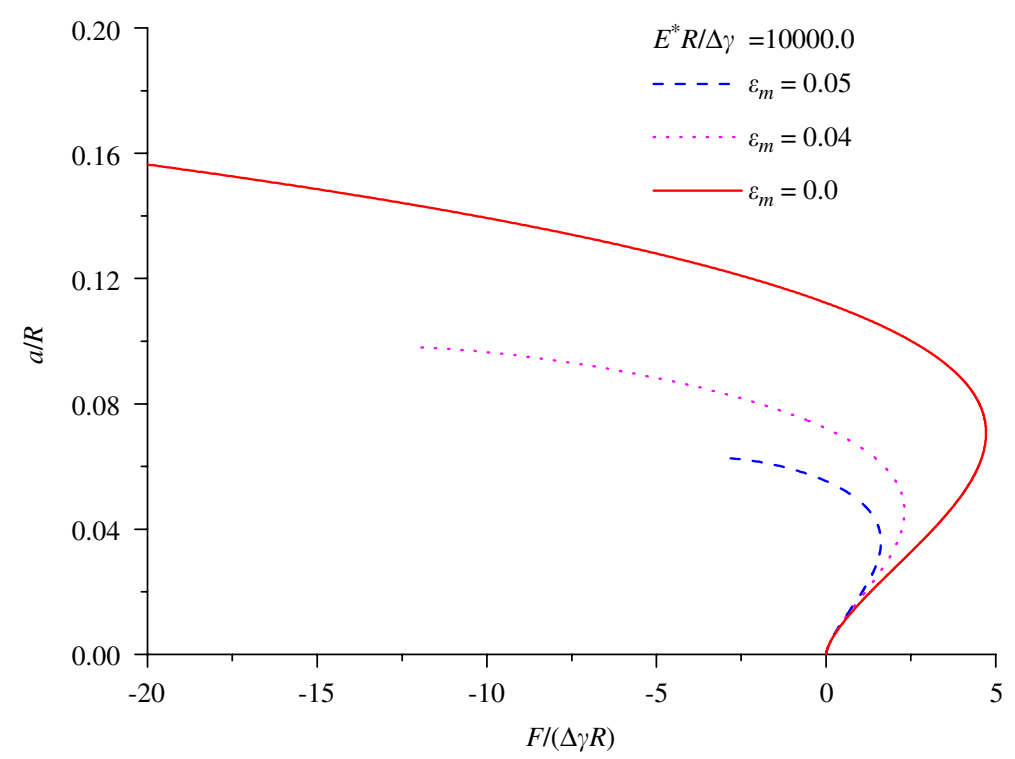

Fig. 5. Pull-off of adhering spheres at a critical force. The normalized contact radius $\tilde{a}=a / R$ is plotted as a function of the normalized pulling force $\tilde{F}=F /(\Delta \gamma R)$ for different values of the mismatch strain $\varepsilon_{m}$. The mismatch strain $\varepsilon_{m}$ is seen to have a significantly effect on the pull-off process. 


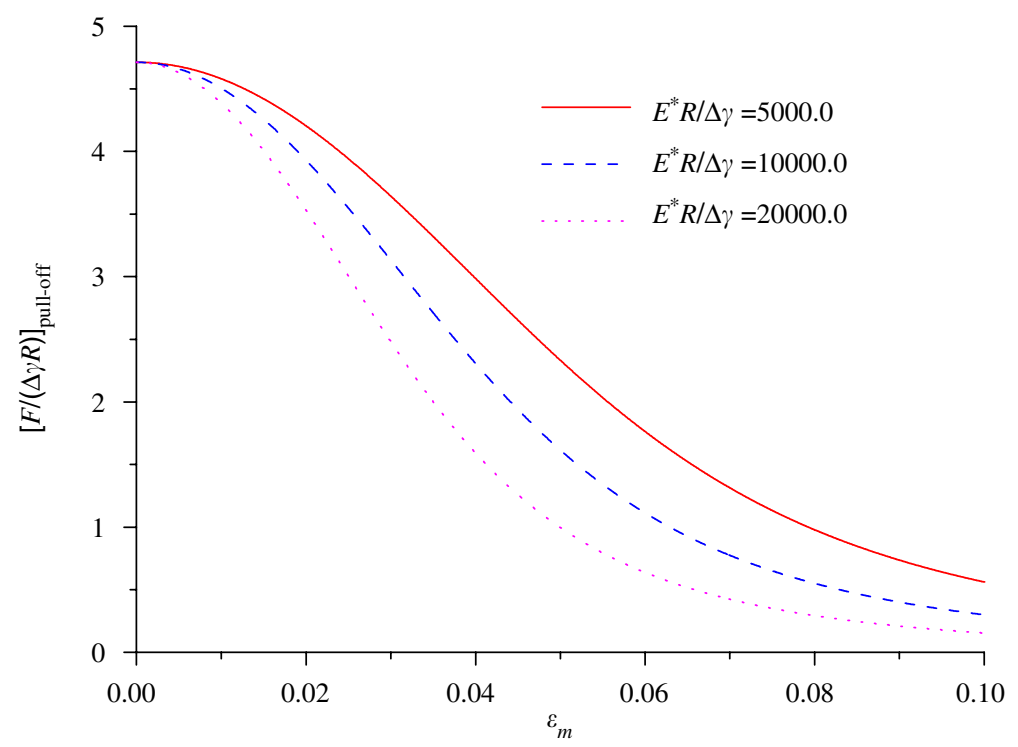

Fig. 6. Effect of the mismatch strain $\varepsilon_{m}$ on the normalized pull-off force $[F /(\Delta \gamma R)]_{\text {pull-off }}$ for different values of the normalized modulus $\tilde{E}=E^{*} R / \Delta \gamma$.

force decreases as the mismatch strain increases. Fig. 6 plots the normalized pull-off force $[\tilde{F}]_{p u l l-o f f}$ as a function of the mismatch strain $\varepsilon_{m}$ for three different values of $\tilde{E}$. In the classical JKR theory, the pull-off force is independent of the Young's modulus (the point of $\varepsilon_{m}=0$ ). This is seen to be no longer true in the presence of a mismatch strain. The pulloff force decreases with increasing modulus at a finite $\varepsilon_{m}$, as shown in Fig. 6.

It can be observed from Fig. 5 that the critical contact radius at pull-off decreases with increasing mismatch strain. Fig. 7 plots the normalized critical radius $[\tilde{a}]_{\text {pull-off }}$ as a function of $\varepsilon_{m}$ for three different values of $\tilde{E}$. The result shows that $[\tilde{a}]_{p u l l-o f f}$ decreases with increasing modulus.

Therefore, the mismatch strain has significant effect on both the critical force and critical contact radius at pull-off. Under a fixed pulling force, there exists a critical mismatch strain at which a pair of adhering spheres is predicted to break apart spontaneously.

\subsection{Adhesion mediated deformation sensor}

The generalized JKR model discussed in the present paper suggests that two adhering objects under thermal fluctuation have an increasing chance to break up in the presence of a mismatch strain induced by environmental forces. Thermal forces tend to break apart any adhering particles and are therefore analogous to the pair of pulling forces considered in the present model. Mismatch strains can arise under changes in environmental pressure or temperature or PH values. The concept of an adhesion mediated deformation sensor is schematically illustrated in Fig. 8.

The total adhesion energy $\Delta U$ of two adhering spheres under a given mismatch strain $\varepsilon_{m}$ (with no pulling force) can be written as

$$
\Delta U=\Delta U_{\text {surface }}-\Delta U_{\text {elastic }},
$$




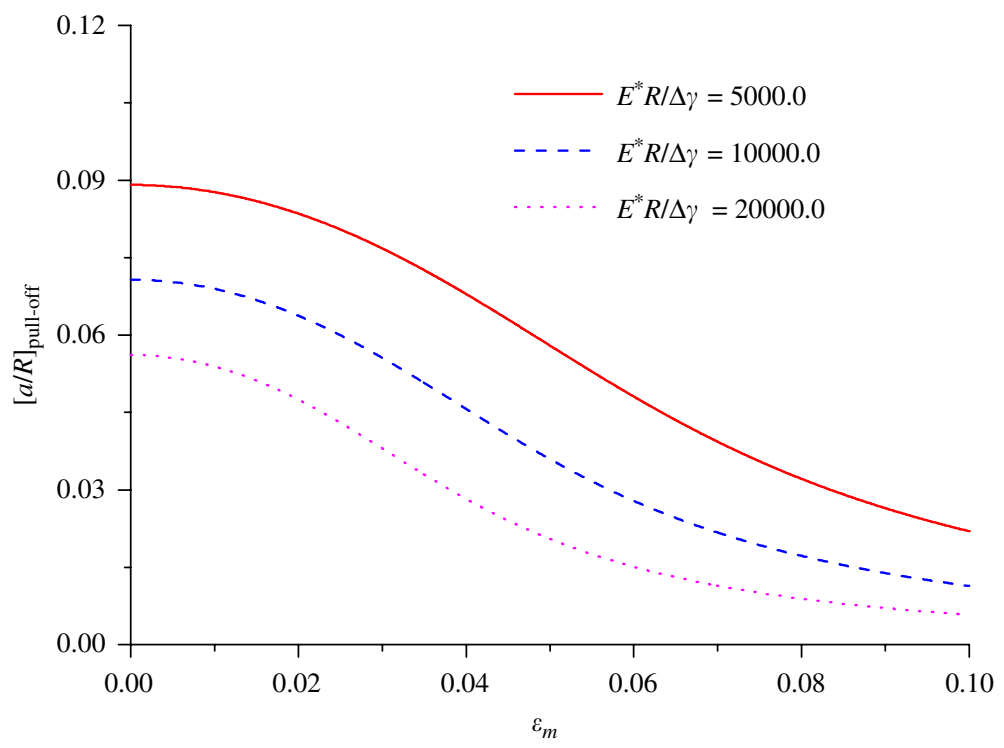

Fig. 7. Effect of the mismatch strain $\varepsilon_{m}$ on the normalized critical contact radius at pull-off $[a / R]_{\text {pull-off }}$ for different values of the normalized modulus $\tilde{E}=E^{*} R / \Delta \gamma$.

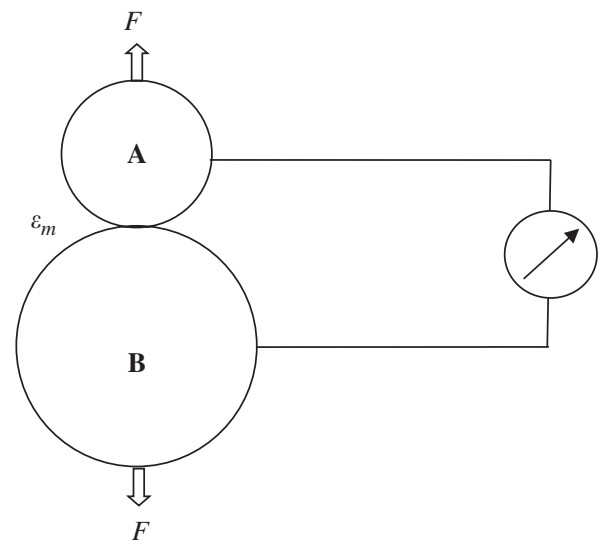

Fig. 8. Schematic of an adhesion mediated deformation sensor. Under a pulling force (e.g., thermal or entropic forces), the adhesion strength and contact area would be sensitive to any environmental forces that can induce a mismatch strain in the bimaterial system.

where $\Delta U_{\text {surface }}$ is the change in surface energies and $\Delta U_{\text {elastic }}$ is the change in elastic energy as the contact is formed. These quantities can be calculated as

$$
\begin{aligned}
& \Delta U_{\text {surface }}=\pi a_{e q}^{2} \Delta \gamma, \\
& \Delta U_{\text {elastic }}=\left.\int_{0}^{a_{e q}} 2 \pi a G\right|_{\varepsilon_{m} \neq 0} ^{F=0} \mathrm{~d} a,
\end{aligned}
$$


where

$$
\left.G\right|_{\varepsilon_{m} \neq 0} ^{F=0}=\frac{K_{\mathrm{I}}^{2}+K_{\mathrm{II}}^{2}}{2 E^{*}}
$$

is the strain energy release rate when $F=0, \varepsilon_{m} \neq 0$ and $a_{e q}$ is given in Eq. (45). In this case, the corresponding stress intensity factors can be obtained from Eqs. (37) and (38) as

$$
\begin{aligned}
& K_{\mathrm{I}}=\frac{2 E^{*} a^{3 / 2}}{3 \sqrt{\pi} R}, \\
& K_{\mathrm{II}}=-2 E^{*} \varepsilon_{m} \sqrt{\frac{a}{\pi}},
\end{aligned}
$$

which, when inserted into Eq. (60), gives

$$
\Delta U_{\text {elastic }}=\left(\frac{4 a_{e q}^{2}}{45 R^{2}}+\frac{4}{3} \varepsilon_{m}^{2}\right) E^{*} a_{e q}^{3} .
$$

Figs. 9 and 10 plot the normalized adhesion energy $\Delta U /\left(K_{B} T\right)$, where $K_{B}$ is the Boltzmann constant and $T=300 \mathrm{~K}$ denotes the room temperature, as a function of $\varepsilon_{m}$ for two different sets of parameter variations. The surface energy is taken to be $2.5 \mathrm{~mJ} / \mathrm{m}^{2}$. Fig. 9 shows that both particle sizes (through the combined radius $R$ ) and their elastic properties (through the combined modulus $E^{*}$ ) have significant effects on the relation between $\Delta U /\left(K_{B} T\right)$ and $\varepsilon_{m}$. The results suggest that a mismatch strain on the order of $10 \%$ and above would be needed to bring the adhesion energy down to the level of thermal

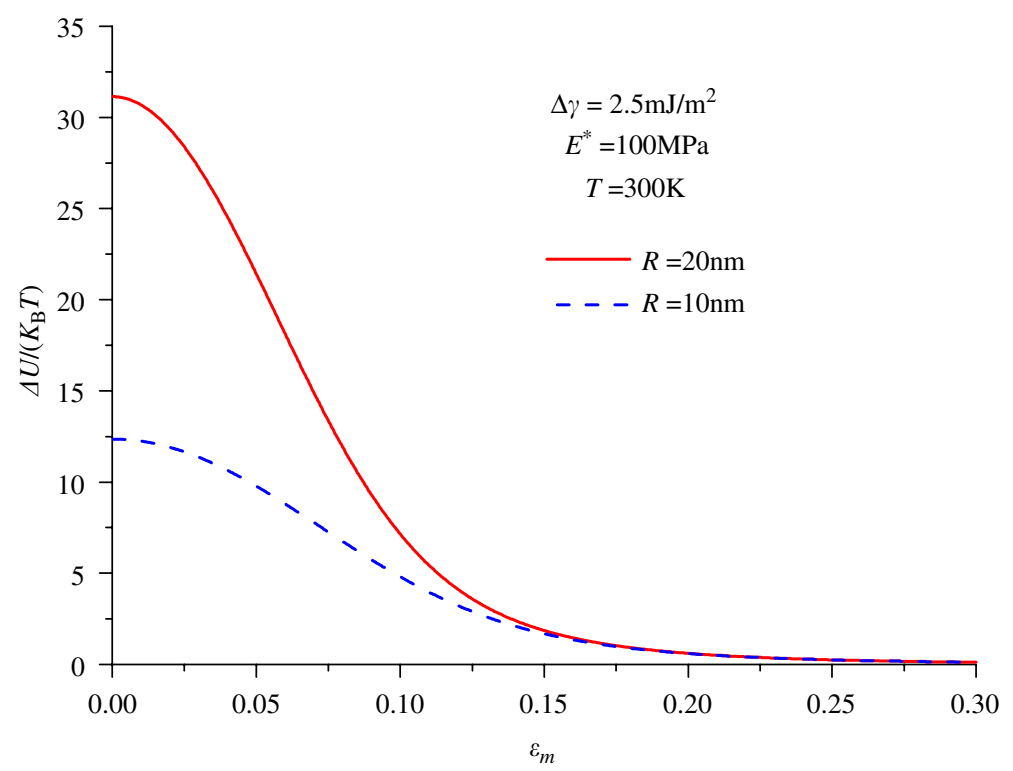

Fig. 9. Variation of the normalized work of adhesion $\Delta U /\left(K_{B} T\right)$ of a pair of particles as a function of the mismatch strain $\varepsilon_{m}$ for two values of the combined radius $R=10$ and $20 \mathrm{~nm}$ and other parameter choices of $\Delta \gamma=2.5 \mathrm{~mJ} / \mathrm{m}^{2}, E^{*}=100 \mathrm{MPa}$ and $T=300 \mathrm{~K}$. 


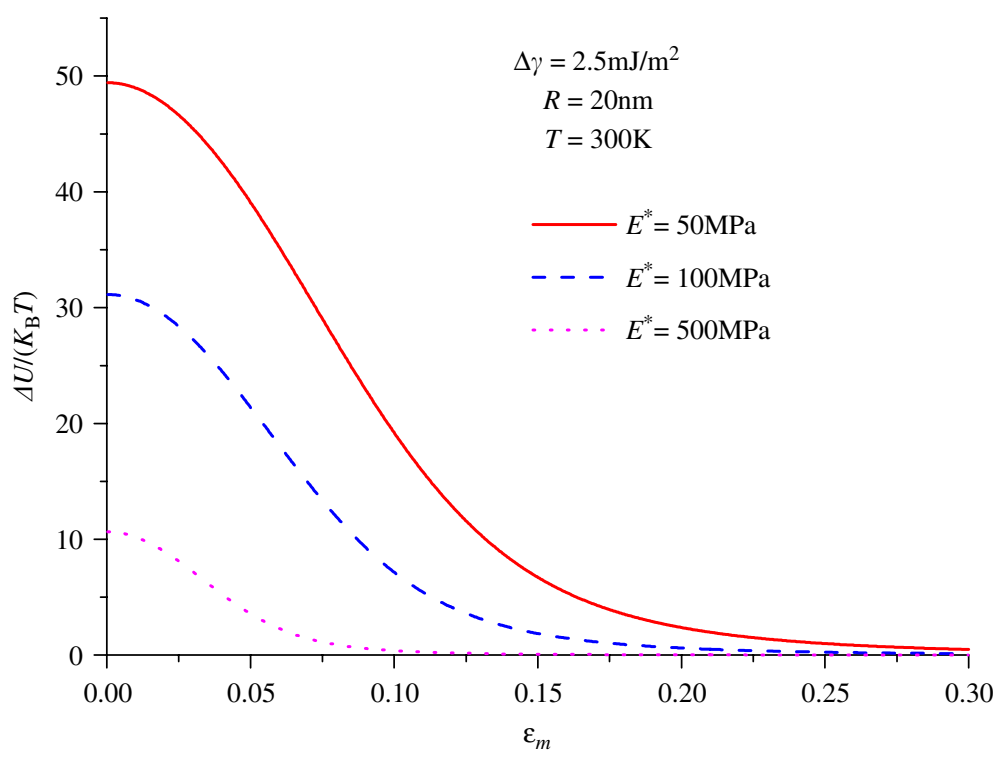

Fig. 10. Variation of the normalized work of adhesion $\Delta U /\left(K_{B} T\right)$ of a pair of particles as a function of the mismatch strain $\varepsilon_{m}$ for three values of the combined modulus $E^{*}=50,100$ and $500 \mathrm{MPa}$ and other parameter choices of $\Delta \gamma=2.5 \mathrm{~mJ} / \mathrm{m}^{2}, R=20 \mathrm{~nm}, T=300 \mathrm{~K}$.

energy $K_{B} T$ so that the adhering particles would spontaneously dissociate under thermal fluctuation.

\section{Acknowledgements}

The work reported here was performed under the auspices of the Max Planck Society when H.G. served as a director at the Max Planck Institute for Metals Research in Stuttgart, Germany, in the last 5 years. S.C. gratefully acknowledges support by a Max Planck Visiting Scientist Fellowship during 2003 and 2005.

\section{References}

Artz, E., Gorb, S., Spolenak, R., 2003. From micro to nano contacts in biological attachment devices. Proc. Natl. Acad. Sci. USA 100, 10603-10606.

Autumn, K., Sitti, M., Liang, Y.C., Peattie, A.M., Hansen, W.R., Sponberg, S., Kenny, T.W., Fearing, R., Israelachvili, J.N., Full, R.J., 2002. Evidence for van der Walls adhesion in gecko setae. Proc. Natl. Acad. Sci. USA 99, 12252-12256.

Barquins, M., 1988. Adherence and rolling kinetics of a rigid cylinder in contact with a natural rubber surface. J. Adhesion 26, 1-12.

Bao, G., 2000. Single-molecule biomechanics: DNA and protein deformation. In: Casey, J., Bao, G. (Eds.), Mechanics in Biology, ASME AMD-vol. 242/BED-vol. 46, pp. 25-35.

Bao, G., 2002. Mechanics of biomolecules. J. Mech. Phys. Solids 50, 2237-2274.

Barthel, E., 1998. On the description of the adhesive contact of spheres with arbitrary interaction potentials. J. Coll. Interface Sci. 200, 7-18.

Barthel, E., Haiat, G., 2002. Approximate model for the adhesive contact of viscoelastic spheres. Langmuir 18, 9362-9370. 
Bischofs, I.B., Schwarz, U.S., 2003. Cell organizing in soft media due to active mechanosensing. Proc. Natl. Acad. Sci. USA 100, 9274-9279.

Carpick, R.W., Agrait, N., Ogletree, D.F., Salmeron, M., 1996. Variation of the interfacial shear strength and adhesion of a nanometer sized contact. Langmuir 12, 3334-3340.

Chaudhury, M.K., Weaver, T., Hui, C.Y., Kramer, E.J., 1996. Adhesion contact of cylindrical lens and a flat sheet. J. Appl. Phys. 80, 30-37.

Chen, S., Gao, H., 2006. Non-slipping adhesive contact of an elastic cylinder on stretched substrates. Proc. Roy. Soc. Lond. A 462, 211-228.

Chu, Y.S., Dufour, S., Thiery, J.P., Perez, E., Pincet, F., 2005. Johnson-Kendall-Roberts theory applied to living cells. Phys. Rev. Lett. 94 (2), 028102.

Dartsch, P.C., Betz, E., 1989. Response of cultured endothelial cells to mechanical stimulation. Basic Res. Cardiol. 84, 268-281.

Dartsch, P.C., Hammerle, H., 1986. Orientation response of arterial smooth muscle cells to mechanical stimulation. Eur. J. Cell Biol. 41, 339-346.

Derjaguin, B.V., Muller, V.M., Toporov, Y.P., 1975. Effect of contact deformations on the adhesion of particles. J. Coll. Interface Sci. 53, 314-326.

Durdurs, J., 1969. Edge-bonded dissimilar orthogonal elastic wedges. J. Appl. Mech. 36, 650-652.

Erdogan, F., 1965. Stress distribution in bonded dissimilar materials with cracks. J. Appl. Mech. 32, $403-410$.

Galbraith, C.G., Sheetz, M., 1998. Forces on adhesive contacts affect cell function. Curr. Opin. Cell Biol. 10, $566-571$.

Gao, H., 1990. Mismatched elastic connections. Int. J. Fract. 45, 131-143.

Gao, H., Yao, H., 2004. Shape insensitive optimal adhesion of nanoscale fibrillar structures. Proc. Natl. Acad. Sci. USA 101, 7851-7856.

Gao, H., Wang, X., Yao, H., Gorb, S., Arzt, E., 2005. Mechanics of hierarchical adhesion structures of gecko. Mech. Mater. 37, 275-285.

Geiger, B., Bershadsky, A., 2002. Exploring the neighborhood: adhesion-coupled cell mechanosensors. Cell 110 , $139-142$.

Glassmaker, N.J., Jagota, A., Hui, C.Y., Kim, J., 2004. Design of biomimetic fibrillar interface: 1. making contact. J. Roy. Soc. Interface 1, 23-33.

Greenwood, J.A., Johnson, K.L., 1981. The mechanics of adhesion of viscoelastic solids. Philo. Mag. 43, 697-711.

Greenwood, J.A., 1997. Adhesion of elastic spheres. Proc. Roy. Soc. Lond. A 453, 1277-1297.

Greenwood, J.A., Johnson, K.L., 1998. An alterative to the Maugis model of adhesion between elastic spheres. J. Phys. D: Appl. Phys. 31, 3279-3290.

Haiat, G., Huy, M.C.P., Barthel, E., 2003. The adhesive contact of viscoelastic spheres. J. Mech. Phys. Solids 51, 69-99.

Haston, W.S., Shields, J.M., Wilkinson, P.C., 1983. The orientation of fibroblasts and neutrophils on elastic substrata. Exp. Cell Res. 146, 117-126.

Howard, J., 2001. Mechanics of Motor Proteins and the Cytoskeleton. Sinauer Associates, Inc., Sunderland, MA.

Huang, S., Ingber, D.E., 1999. The structural and mechanical complexity of cell-growth control. Nat. Cell. Boil. 1, E131-E138.

Huber, G., Gorb, S., Spolenak, R., Artz, E., 2005. Resolving the nanoscale adhesion of invidual gecko spatulae by atomic force mocroscopy. Biol. Lett. 1, 2-4.

Hui, C.Y., Baney, J.M., Kramer, E.J., 1998. Contact mechanics and adhesion of viscoelastic spheres. Langmuir 14, 6570-6578.

Hui, C.Y., Glassmaker, N.J., Tang, T., Jagota, A., 2004. Design of biomimetic fibrillar interface: 2. mechanics of enhanced adhesion. J. Roy. Soc. Interface 1, 35-48.

Iba, T., Sumpio, B.E., 1991. Morphological response of human endothelial cells subjected to cyclic strain in vitro. Microvasc. Res. 42, 245-254.

Johnson, K.L., 1985. Contact Mechanics. Cambridge University Press, Cambridge.

Johnson, K.L., Greenwood, J.A., 1997. An adhesion map for the contact of elastic spheres. J. Coll. Interface Sci. 192, 326-333.

Johnson, K.L., Kendall, K., Roberts, A.D., 1971. Surface energy and the contact of elastic solids. Proc. Roy. Soc. Lond. A 324, 301-313.

Kendall, K., 1975. The effects of shrinkage on interfacial cracking in a bonded laminate. J. Phys. D: Appl. Phys. 8, $1722-1732$. 
Kim, K.S., McMeeking, R.M., Johnson, K.L., 1998. Adhesion, slip cohesive zone and energy fluxes for elastic spheres in contact. J. Mech. Phys. Solids 46, 243-266.

Lin, Y.Y., Hui, C.Y., Baney, J.M., 1999. Viscoelastic contract, work of adhesion and the JKR technique. J. Phys. D: Appl. Phys. 32, 2250-2260.

Lo, C.-M., Wang, H.-B., Dembo, M., Wang, Y-L., 2000. Cell movement is guided by the rigidity of the substrate. Biophys. J. 79, 144-152.

Lorz, B., Simson, R., Nardi, J., Sackmann, E., 2000. Weakly adhering vesicles in shear flow: tanktreading and anomalous lift force. Europhys. Lett. 51, 468-474.

Maugis, D., 1992. Adhesion of spheres: The JKR-DMT transition using a Dugdale model. J. Coll. Interface Sci. 150, 243-269.

Maugis, D., Barquins, M., 1978. Fracture mechanics and the adherence of viscoelastic bodies. J. Phys. D 11, 1989-2023.

Morrow, C., Lovell, M., Ning, X., 2003. A JKR-DMT transition solution for adhesive rough surface contact. J. Phys. D: Appl. Phys. 36, 534-540.

Muller, V.M., Yushenko, V.S., Derjaguin, B.V., 1980. On the influence of molecular forces on the deformation of an elastic sphere and its sticking to s rigid contact. J. Coll. Interface Sci. 77, 91-101.

Neidlinger-Wilke, C., Wilke, H.J., Claes, L., 1994. Cyclic stretching of human osteoblasts affects proliferation and metabolism: a new experimental method and its application. J. Orthop. Res. 12, 70-78.

Persson, B.N.J., 2003. On the mechanism of adhesion in biological systems. J. Chem. Phys. 118 (16), 7614-7621.

Rice, J.R., 1965. Plane problems of crack in dissimilar media. J. Appl. Mech. 32, 418-423.

Robbe-Valloire, F., Barquins, M., 1998. Adhesive contact and kinetics of adherence between a rigid cylinder and an elastomeric solid. Int. J. Adhesion Adhesives 18, 29-34.

Roberts, A.D., Thomas, A.G., 1975. The adhesion and friction of smooth rubber surfaces. Wear 33, 45-64.

Savkoor, A.R., Briggs, G.A.D., 1977. The effect of a tangential force on the contact of elastic solids in adhesion. Proc. Roy. Soc. Lond. A 356, 103-114.

Schwarz, U.D., 2003. A generalized analytical model for the elastic deformation of an adhesive contact between a sphere and a flat surface. J. Coll. Interface Sci. 261, 99-106.

Sneddon, I.N., 1951. Fourier transformations. McGraw-Hill, New York.

Spence, D.A., 1968a. Self similar solutions to adhesive contact problems with incremental loading. Proc. Roy. Soc. Lond. A 305, 55-80.

Spence, D.A., 1968b. A Wiener-Hopf equation arising in elastic contact problems. Proc. Roy. Soc. Lond. A 305, $81-92$.

Spolenak, R., Gorb, S., Gao, H., Artz, E., 2005. Effects of contact shape on the scaling of biological attachments. Proc. Roy. Soc. Lond. A 461, 305-319.

Takemasa, T., Sugimoto, K., Yamashita, K., 1997. Amplitude-dependent stress fiber reorientation in early response to cyclic strain. Exper. Cell Res. 230, 407-410.

Wang, H.C., Wallace, I., Raymond, B., Edward, S.G., 1995. Cell orientation response to cyclically deformed substrates: experimental validation of a cell model. J. Biomech. 28, 1543-1552.

Westmann, R.A., 1965. Asymmetric mixed boundary-value problems of the elastic half-space. J. Appl. Mech. 32, 411-417.

Wong, J.Y., Velasco, A., Rajagopalan, P., Pham, Q., 2003. Directed movement of vascular smooth muscle cells on gradient-compliant hydrogels. Langmuir 19, 1908-1913.

Yao, H., Gao, H., 2006. Mechanics of robust and releasable adhesion in biology: bottom-up designed hierarchical structures of gecko. J. Mech. Phys. Solids 54, 1120-1146.

Zhu, C., Bao, G., Wang, N., 2000. Cell mechanics: mechanical response, cell adhesion, and molecular deformation. Annu. Rev. Biomed. Eng. 2, 189-226. 